\title{
Role of response imagery on stimulus recognition*
}

\author{
B. CHARLES TATUM and HENRY C. ELLIS† \\ Lniversity of New Mexico, Albuquerque, New Mexico 87106
}

\begin{abstract}
This experiment investigated the role of response imagery on recognition of stimulus terms following associative training. Ss learned to associate 12 high-imagery or low-imagery responses with trigram stimuli by the study-associative matching procedure. Half the pairs were consistently paired and half were re-paired anew on each trial. Following associative training. Ss were given a stimulus recognition and an associative matching task. The results indicated that the probability of stimulus recognition, given correct associative matching, was greater when the responses were high-imagery rather than low-imagery. In contrast, no reliable response imagery effects were found for the probability of stimulus recognition. given incorrect associative matching. It was argued that the locus of the response imagery effect was primarily during retrieval rather than during encoding.
\end{abstract}

This experiment investigated the influence of response-term imagery on stimulus recognition following paired-associate (PA) training. The specific problem concerns whether response imagery can enhance the recognizability of a stimulus and whether the locus of this effect is principally during the stimulus encoding stage of PA learning or during retrieval at the time of stimulus recognition testing. To investigate this problem, contingencies relating stimulus recognition and associative matching were examined.

The problem bears on the more general issue of response-dependent stimulus encoding (cf. Ellis, in press). It has been shown that the manner in which $S$ encodes stimuli in a PA task can depend upon properties of the response terms employed and that response terms which are "representative" of the stimuli will lead to more rapid and efficient encoding of visual form (Ellis, 1968; Ellis \& Daniel, 1971) and verbal stimuli (Ellis \& Shumate. in press). Ellis and Daniel have proposed that response factors exert their principal influence during the stimulus selection/encoding stage of associative learning as distinct from retrieval processes operating at the time of recognition testing. Moreover, they proposed that response-dependent stimulus encoding is the result of at least three factors. including the use of responses which are in some sense representative of the stimuli. which direct S's attention to certain critical or distinctive features of the stimuli. and/or which encourage the use of image-like mediators during associative learning. Although there is considerable evidence for the operation of the first two factors (e.g.. Ellis. 1968: Ellis \& Daniel. 1971: Ellis \& Muller. 1964: Ellis \& Shumate. in press), there is essentially no

*This research was supported by a National Defense Education Act graduate fellowship to the first author and by National Science Foundation Grant GB-27413X to the second author.

$\div$ Requests for reprints should be sent to Henry C. Ellis. Department of Psychology. University of New Ifexico, Albuquerque. New Mexico 87106. evidence directly relevant to the third factor. Evidence for this third factor would be demonstrated by showing that stimulus recognition is enhanced when the response terms are high-imagery (I) as opposed to low-I, since high-I responses should encourage $S$ to use image-like mediators more frequently during association formation.

To determine whether high-I responses do indeed encourage $\mathrm{S}$ to use image mediators during associative learning, the present study compared stimulus recognition performance following two types of $\mathrm{PA}$ training. One type (Type C) was simply the standard PA procedure in which $\mathrm{S}$ consistently saw the same response with a given stimulus across trials. For the other type (Type R), the stimuli were re-paired with new responses on each successive trial. If $S$ is forming stronger image mediators with the Type $C$ pairs than with the Type $R$ pairs, then his stimulus recognition performance should be superior to that of $\mathrm{Ss}$ in the Type $\mathrm{R}$ procedure, because the Type $\mathrm{R}$ procedure should mitigate against the formation of strong image mediators.

In contrast to the stimulus selection/encoding interpretation is the view that response imagery primarily effects retrieval processes operating at the time of the test for stimulus recognition. This view contends that the facilitative effect of response imagery on stimulus recognition results from the response providing $S$ with a reliable cue for retrieving some stored representation of the stimulus. Furthermore, it predicts a dependency between S's ability to match correctly a response to its stimulus mate and his ability to recognize that stimulus correctly. Specifically. according to a retrieval hypothesis, high-I responses will lead to superior stimulus recognition over low-I responses only when the response is accessible to $S$ and thus presumably can serve as a retrieval cue. Therefore. response imagery should influence stimulus recognition only when $\mathrm{S}$ can match correctly the response with the stimulus at the time of the recognition test. If $S$ fails to match correctly the response with the stimulus. then the response is obviously inaccessible as a retrieval cue and no response 
imagery effects should emerge. It is this contingency between associative matching and stimulus recognition performances which distinguishes between the encoding hypothesis and a retrieval hypothesis. Since the encoding hypothesis emphasizes processes which occur during original learning and not during testing, this hypothesis predicts that response imagery effects on stimulus recognition will not be contingent upon the outcome of associative matching at the time of the stimulus recognition test.

\section{METHOD}

\section{Design and Materials}

The Ss were 40 undergraduate student volunteers from the Lniversity of New Mexico. The experiment conformed to a mixed design with two between-S and two within-S factors. Two levels of a list factor (Lists 1 and 2) and two types of response terms (high-I and low-I) were the between-S factors. Two types of pairs (Type $C$ and Type $R$ ) and trials accounted for the within-S factors.

The experiment employed a modification of the study-test procedure which involves the sequence of study-test trials with test trials involving both stimulus recognition and associative matching. Each $\mathrm{S}$ received six study trials. Half the Ss were assigned randomly to study 12 trigram high-l word pairs, and the remaining half studied 12 trigram low-I word pairs. The trigram stimuli were CCCs with an average association value of $67 \%$ according to witmer (1935). The response terms were either high-I nouns or low-I nouns as rated by Paivio, Yuille, and Madigan (1968). The average I values for the high-I and low-I nouns were 6.29 and 3.79 , respectively. Average meaningfulness (m) values for the high-I and low-I responses were roughly comparable at an intermediate level (5.87 and 5.57. respectively), as scaled by Paivio. Yuille, and Madigan (1968). Since concreteness correlates highly with $I$ value and is theoretically linked to I value (Paivio. 1969), no control for concreteness was made. All of the responses were three- to five-letter words. Examples of pairs with high-I responses are GRD-DRESS and FRN-HARP. Examples of pairs with low-I responses are GRD-DRAMA and FRN-HOPE.

Of the 12 trigram high-I response (or trigram low-I) pairs, 6 were designated as Type $C$ and 6 as Type $R$. The Type $C$ pairs were consistently paired throughout learning, whereas the 6 Type $R$ pairs were re-paired anew on each study trial. The same 6 responses (high-I or low-I) were used for both Type $R$ and Type $C$ pairs. The Type $R$ pairs were included to determine if changing the responses on each study trial had any effect on stimulus recognition performance. Conceivably, the Type R pairs may lead to a decrease in stimulus recognition performance relative to the Type $\mathrm{C}$ pairs when the responses are high-I, since this procedure should mitigate against the formation of strong image mediators.

Each $\mathrm{S}$ learned both Type $\mathrm{C}$ and Type $\mathrm{R}$ pairs in a mixed list. In addition, two counterbalanced lists (Lists 1 and 2) were constructed such that those trigrams entering into a Type $C$ pair in one list would enter into a Type $R$ pair on a second list, and vice versa. Of the $20 \mathrm{Ss}$ assigned to learn trigram high-I response pairs. 10 were assigned randomly to List 1 and 10 to List 2 . Similarly, of the $20 \mathrm{Ss}$ assigned to learn trigram low-I pairs, 10 were assigned randomly to List 1 and 10 to List 2.

\section{Recognition and Matching Tests}

On each of the six recognition test trials, $\mathrm{S}$ was presented 12 trigram pairs. One of the trigrams in the pair was an old item cone that $s$ saw during study) and the other was a new item (distractor). Of the 12 old trigrams, 6 were from Type $C$ and 6 were from Type R pairs.

The 12 new items were constructed to maximize their similarity to the old items to make the task of sufficient difficulty and thus avoid possible ceiling effects. For each old trigram, distractor trigrams were constructed by taking different combinations of the three letters. Only five such combinations were possible (the sixth combination being the study trigram itself). Therefore. a sixth distractor was constructed by replacing the last letter of the study trigram with a high-frequency consonant. For each of the old trigrams, the consonant chosen for replacement was one which had a high frequency of occurrence in conjunction with the middle letter of the old trigram, as determined by the bigram frequencies reported in Underwood and Schulz (1960). A particular final consonant was used only once for each distractor. Six different distractor trigrams were needed, of course, because a new distractor was paired with the old trigram in each recognition test.

To each of the trigram pairs, $S$ first had to respond with a recognition response and then had to match the high-I or low-I response paired with that trigram.

\section{Procedure}

Six different dittoed sheets were prepared for each of the six test trials. Each sheet contained a list of 12 trigram pairs, one item being old and the other new, with the position of each trigram in a pair randomized. In addition, the ordinal position of the pairs was randomized. To the right of each pair, space was provided to write the appropriate high-I or low-I response.

Each S was told that study and test trials would alternate regularly, beginning with a study trial. During the study trials, $S$ was required to view and study the 12 trigram high-I or trigram low-I pairs. The 12 pairs were presented on a Stowe memory drum at a $2-\mathrm{sec}$ rate. There were six random orders of presentation for the study-trial items.

On each test trial, $S$ was given one of the dittoed sheets at random. The $S$ was required to place the sheet between two pieces of cardboard. The top piece of cardboard had a slot sufficiently large to view each trigram pair separately. Space to the right of the pair was provided to write the appropriate response. The $S$ was instructed to align the first trigram pair in the view slot and then check the trigram which he believed to be old. Following recognition. $S$ was then required to write the response (depending upon which condition of the experiment $S$ was assigned) which was paired with that trigram on the immediately preceding study trial. The $\mathrm{S}$ was required to guess if he could not remember the correct response.

Since it was possible that the high-I response terms were more available than the low-I response terms, each S was given a card which contained a list of either all the high-I or all the low-I response terms (depending upon which condition of the experiment $S$ was assigned) presented on the study trials. The words were arranged on the card in a random fashion. and $S$ was allowed to use the card during test trials. Thus, the procedure was an associative matching rather than a recall test. Ellis and Tatum (in press) have shown that the results are comparable regardless of whether associative matching or recall procedures are used. When $S$ had made both a stimulus (trigram) recognition response and an associative match to the first trigram pair. he then proceeded to the next trigram pair and repeated the above procedure. The $S$ had $6 \mathrm{sec}$ for each trigram, and the test trial was completed when $S$ had responded to all 12 trigram pairs on the sheet.

The $\mathbf{S}$ was told that there would be two types of trigrams on the study trials (Type $C$ and Type $R$ ). The intertrial interval was approximately $5 \mathrm{sec}$. The $S$ was not explicitly told how many study and test trials there would be. The $E$ emphasized to $S$ that the important aspect of the experiment was to learn to pair. or associate the items on each study trial. 
Fig. 1. Probability of correct stimulus recognition over trial blocks given correct and incorrect associative matching of high-I and low-I response terms.
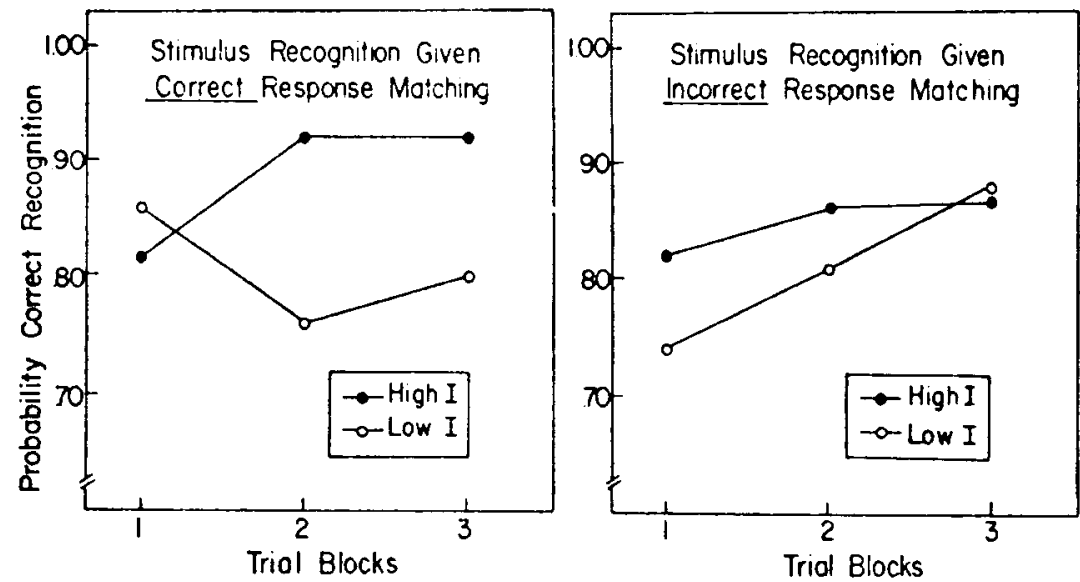

\section{RESULTS}

\section{Type $\mathrm{C}$ vs Type $\mathrm{R}$}

The results indicated that there were no differences in stimulus recognition performance for the Type $\mathrm{C}$ and Type $\mathrm{R}$ pairs, as supported by a nonsignificant main effect for pair type ( $C$ vs $R$ ) $[F(1.36)=.87 . p>.05]$. Moreover. the Pair Type by Response Type (high-I vs low-I) interaction was not reliable $[\mathrm{F}(1,36)=1.63$. $\mathrm{p}>.05]$. This finding indicates that changing the response term on each trial (Type $R$ ), which mitigated against the formation of image mediators, did not retard stimulus recognition relative to Type $C$ pairs.

\section{Associative Matching}

It is sufficient to say that across all conditions. $\mathrm{S}$ could correctly match the response with the stimuli at a level significantly exceeding chance $[t(9)=5.71$, $\mathrm{p}<.001]$. This finding simply indicates that Ss were learning to associate the responses with the stimuli during associative training. Moreover. degree of learning does not differ for any of the conditions of the experiment as evidenced by nonsignificant main effects for response imagery (high-I vs low-I) and pair type (C vs R) $[F(1.36)=1.52 . p>.05$. and $F(1.36)=.82 . p>05$. respectively] and a nonsignificant Imagery by Pair Type interaction $[F(1.36)=.004, p>.05]$.

\section{Stimulus Recognition-Associative Matching Contingency}

Conditional probabilities involving stimulus recognition and associative matching were calculated in order to determine the locus of any response imagery effects on stimulus recognition. Figure 1 portrays the probability of correct stimulus recognition over blocks of trials with response type (high-I vs low-I) as the parameter. The left panel of Fig. 1 shows the probability of correct stimulus recognition given a correct matching of the response with the stimulus. The right panel of
Fig. 1 shows the probability of correct stimulus recognition given an incorrect response match. Both panels are based on the Type $\mathrm{C}$ data only.

Inspection of Fig. 1 reveals that the probability of stimulus recognition given a correct response match (left panel) is higher when the response terms are high-I rather than low-I for the last two trial blocks. Although an analysis of variance for this measure failed to show a significant main effect for response type $[F(1,36)=$ 2.94. $\mathrm{p}>.05$ ] the Response Type by Trial Block interaction was reliable $[F(2,72)=5.74 . p<.01]$. Inspection of the right panel also suggests an interaction with respect to the probability of stimulus recognition given an incorrect response match. However, neither the main effect of response type nor the Response Type by Trial Block interaction were significant for this measure $[F(1.38)=1.11, p>.05$. and $F(2.76)=.83 . p>.05$. respectively].

The degrees of freedom for recognition given correct associative matching (left panel) are different from those of recognition given incorrect associative matching (right panel) because some $\mathrm{Ss}$ on some trials failed to match correctly any of the responses. and thus these data were excluded from the analysis. This could conceivably produce a source of bias, in that the "slow learners" could contribute more to the data in the right than in the left panel of Fig. 1. This is highly unlikely, however, since most of the excluded data was from the first trial block (four Ss) and only one $\mathrm{S}$ each was excluded from the second and third blocks of the left panel. If there were a bias. it would be most evident on the first trial block. As can be seen from Fig. 1. the differences in the two panels are most pronounced on the last two trial blocks where any bias is minimal. If there were, in fact. any bias, it would have worked against. not for. the predicted results.

The results of the conditional probability analysis indicate that there is a contingency between stimulus recognition and associative matching performance as predicted by a retrieval hypothesis. The analysis reveals that response imagery is correlated with stimulus recognition but significantly so only when the response 
is accessible in the sense of being correctly matched with its stimulus mate at the time of the recognition test.

\section{DISCUSSION}

The results of the present study show that response imagery effects the recognizability of a stimulus following PA training. Specifically, the results revealed a positive relationship between stimulus recognition and response imagery. Moreover, this effect was contingent upon a correct associative match of the responses with the stimulus, a finding which supports a retrieval view of the effects of response imagery.

There are basically two positions concerning the locus of the response imagery effect on stimulus recognition. The first view is an encoding hypothesis which contends that stimulus recognition following associative training is directly enhanced as a function of response imagery. The encoding may be either a representation of a stimulus-response unit produced by image-like mediators or simply an encoding of the stimulus per se. It seems unlikely, however. that Ss employed image mediators differentially during associative training. If so, then stimulus recognition for the Type $\mathrm{R}$ pairs should have been retarded relative to the Type $C$ pairs, since the Type $\mathrm{R}$ pairs would mitigate against the development of image mediators.

The second view of response imagery effects on stimulus recognition is a retrieval hypothesis. This view, while not denying that response imagery may effect the manner in which $\mathrm{S}$ encodes the stimulus, contends that enhanced stimulus recognition following associative training with high-I (as opposed to low-I) response terms is the result of retrieval processes operating at the time of the recognition test. Specifically, high-I responses provide $\mathrm{S}$ with a more reliable cue for retrieving some stored encoding of the stimulus than do low-I responses. Since the response term must be accessible to $S$ if it is to serve as an effective retrieval cue, the fact that response imagery affects stimulus recognition primarily when $S$ successfully matches the response to the stimulus is consistent with a retrieval hypothesis. Obviously, the differences obtained in the present study were not large in any absolute sense. This is probably due to the procedure employed in that $S$ was not given explicit imagery instructions. If $S$ had been instructed on how best to use the high-I responses, much larger effects would probably have been obtained.

Although the data indicate that the effect of response imagery on stimulus recognition is principally on the retrieval stage rather than the encoding stage, this cannot be taken to mean that retrieval per se is more important or more fundamental than encoding. Certainly $\mathrm{S}$ cannot retrieve something that has not been encoded. The lack of a Type $\mathrm{C} /$ Type $\mathrm{R}$ difference indicates that response imagery does not influence the development of image mediators differentially. This finding does not, however, preclude the possibility that response imagery can influence the encoding of the stimulus per se. Thus, the issue is whether a high-imagery response term, after possibly influencing S's encoding of the stimulus during associative learning. need be accessible at the time of the recognition test to aid additionally in the retrieval process. If such is the case, and the results indicate that it is, then response imagery has its principal effect on retrieval, since any possible influence on stimulus encoding is not sufficient. Although Paivio (1969) emphasizes the role of stimulus imagery rather than response imagery in PA learning, he acknowledges that either a high-I stimulus or a high-I response can serve as a retrieval cue for the other, provided that it is explicitly presented by E (Paivio, 1971, Chap. 8 and 10). The present study is obviously consistent with Paivio's view. The present finding contrasts with previous studies (e.g., Ellis \& Daniel, 1971; Ellis \& Shumate, in press) which have shown that the response term does not need to be accessible during recognition testing in order to influence stimulus recognition performance. In these studies, the variable of response representativeness, as distinct from imagery, was shown to have its principal effect on the encoding stage.

\section{REFERENCES}

Ellis. H. C. Transfer of stimulus predifferentiation to shape recognition and identification learning: Role of properties of verbal labels. Journal of Experimental Psychology, 1968. 78, $401-409$.

Ellis, H. C. Stimulus encoding processes in human learning and memory. In G. H. Bower (Ed.), The psychology of learning and motivation. Vol. 7. New York: Academic Press. in press.

Ellis, H. C., \& Daniel, T. C. Verbal processes in long-term stimulus-recognition memory. Journal of Experimental Psychology, 1971, 90, 18-26.

Ellis, H. C., \& Muller, D. G. Transfer in perceptual learning following stimulus predifferentiation. Journal of Experimental Psychology, 1964, 68, 388-395.

Ellis, H. C., \& Shumate, E. C. Encoding effects of response belongingness and stimulus meaningfulness on recognition memory of trigram stimuli. Journal of Experimental Psychology, in press.

Ellis, H. C., \& Tatum, B. C. Stimulus encoding and the relationship between stimulus recognition and association formation. Journal of Verbal Leaming \& Verbal Behavior, in press.

Paivio, A. Mental imagery in associative learning and memory. Psychological Review, 1969, 76, 241-267.

Paivio, A. Imagery and verbal processes. New York: Holt, Rinehart \& Winston, 1971

Paivio. A., Yuille, J. C., \& Madigan. S. A. Concreteness, imagery and meaningfulness values for 925 nouns. Journal of Experimental Psychology, 1968, 76(Monogr. Suppl. 1), 1.25.

Underwood, B. J., \& Schulz, R. W. Meaningfuiness and verbal learning. Philadelphia: Lippincott. 1960.

Witmer, T. R. The association value of three-place consonant syllables. Journal of Genetic Psychology, 1935, 47, 337-360.

(Received for publication September 25, 1972; revision received December 8.1972. ) 